QUARTERLY OF APPLIED MATHEMATICS

VOLUME LXIV, NUMBER 3

SEPTEMBER 2006, PAGES 433-445

S 0033-569X(06)01013-2

Article electronically published on June 13, 2006

\title{
THE EXACT REGION OF OSCILLATION FOR A FIRST ORDER NEUTRAL DIFFERENTIAL EQUATION WITH DELAYS
}

\author{
BY \\ SUI SUN CHENG (Department of Mathematics, Tsing Hua University, Hsinchu, Taiwan 30043, \\ Republic of China) \\ AND \\ YI-ZHONG LIN (Department of Mathematics, Fujian Normal University, Fuzhou, Fujian 350007, \\ People's Republic of China)
}

Abstract. The theory of envelopes is applied to yield the exact geometric region of oscillation for a class of first order neutral differential equation with delays. As examples, we show that the convex region of oscillation yield oscillation criteria that are sharp.

1. Introduction. When delays appear in additional terms involving the derivative of the unknown function in a differential equation, we are then dealing with a neutral type differential equation. In recent years, such equations have been the subject of numerous investigations. Indeed, a search conducted on MathSciNet of the American Mathematical Society leads to over 400 recent publications related to the oscillation properties alone. Even if the order of the equations is restricted to 1, there are still over 70 publications in the single area of oscillation.

Yet for very simple linear neutral differential equations with constant coefficients, "explicit and characterizing" conditions for all solutions to oscillate are rare. Indeed, a search of the literature results in only several studies. In particular, in [1], the neutral differential equation

$$
x^{\prime}(t)+\tilde{p} x^{\prime}(t-\tau)+q_{1} x(t)+q_{2} x(t-\sigma)=0, \tilde{p}, q_{1}, q_{2} \in R, \tau, \sigma>0,
$$

with two delays is studied. By means of the inverse functions of restrictions of the function $f(x)=x\left(e^{x}+A\right)$ on various intervals [2], 'algebraic' necessary and sufficient conditions are found for all its solutions to oscillate. In [3], the neutral differential equation

$$
x^{\prime}(t)+\tilde{p} x^{\prime}(t-\tau)+q_{1} x(t)+\tilde{q} x(t-\tau)+q_{3} x(t+\sigma)=0, \tilde{p}, \tilde{q}, q_{1}, q_{3} \in R, \tau, \sigma>0,
$$

with one delay and one advancement is studied. By means of the theory of envelopes, a complete set of 'geometric' necessary and sufficient conditions are found for all its

Received March 11, 2005.

2000 Mathematics Subject Classification. Primary 34C10.

(C)2006 Brown University

Reverts to public domain 28 years from publication 
solutions to oscillate. Clearly, the equation

$$
x^{\prime}(t)-p x^{\prime}(t-\tau)+q x(t-\sigma)=0, p, q \in R, \tau, \sigma>0,
$$

is a special case of (1) (but a special case of (2) only if $\tau=\sigma$ ), and hence the oscillation criterion for (11) is applicable to it. As we will see, however, the 'geometric' method of envelopes in [3] can be applied and give rise to convex 'regions of oscillation' in the $(p, q)$-plane. The nice convexity property will then yield sharp and explicit oscillation criteria for (3), while conditions in [1] are 'algebraic' in nature, and it seems difficult to derive similar oscillation criteria from them.

Although the method of envelopes [4 has been employed by the authors in several studies, for the sake of completeness we briefly go through the essentials of the theory of envelopes which are needed in the sequel. Given a family of curves, a curve that is tangent at each of its points to some curve of the family is called the envelope of the given family. For a family of plane curves given by an equation of the form

$$
h(\lambda ; u, v)=0,
$$

where $u$ and $v$ are the coordinates of the points in the plane and $\lambda$ is a parameter, every point $(x, y)$ of its envelope satisfies the pair of equations

$$
h(\lambda ; x, y)=0 ; h_{\lambda}(\lambda ; x, y)=0 .
$$

We remark that this pair of equations determine a discriminant curve of the family which may contain points other than those which belong to the envelope. However, when the family of plane curves do not have "singular points", such points cannot exist. As we will see below, the families of plane curves in our paper contain nothing more than straight lines, and hence they do not have singular points.

Let $\mu=\max \{\tau, \sigma\}$. We say that $x=x(t)$ is a solution of (3) provided that there exists $t_{0}$ such that $x \in C\left(\left[t_{0}-\mu, \infty\right), R\right), x(t)-p x(t-\tau) \in C^{1}\left[t_{0}, \infty\right)$ and equation (3) holds for $t \geq t_{0}$. As is customary, a solution is called oscillatory if it has arbitrarily large zeros and non-oscillatory otherwise. It is well known that every solution of (3) oscillates if, and only if, the characteristic equation

$$
\lambda-p \lambda e^{-\tau \lambda}+q e^{-\sigma \lambda}=0
$$

has no real roots.

We remark that oscillation problems of delay differential equations such as (3) arise from the observation of swaying of ships due to delay in control mechanisms, from global stability of equilibrium states in population dynamics, global asymptotic behavior of models of hematopoiesis, etc. (see e.g. [6]). Therefore, our investigations in this paper will likely find applications in areas related to asymptotic behaviors of functional differential equations.

2. Exact region of oscillation. We will assume throughout the rest of our discussions that the numbers $p, q, \tau, \sigma$ in (3) satisfy $p, q \in R$ and $\tau, \sigma>0$.

We will need the properties of the parametric function

$$
(x(\lambda), y(\lambda))=\left(e^{\tau \lambda} \frac{1+\sigma \lambda}{1+(\sigma-\tau) \lambda}, e^{\sigma \lambda} \frac{\tau \lambda^{2}}{1+(\sigma-\tau) \lambda}\right),
$$


which is defined everywhere if $\sigma=\tau$ and is defined for $\lambda \neq-1 /(\sigma-\tau)$ if $\sigma \neq \tau$. Since $\sigma$ and $\tau$ are involved, we need to consider different cases. Before going into detail, we first show the following for motivation and later use.

LEMma 1. When restricted to $\lambda \in(-1 / \sigma, 0)$, the parametric function in (5) traces out a curve $C_{(-1 / \sigma, 0)}$ which connects the point $(x(-1 / \sigma), y(-1 / \sigma))=(0,1 /(e \sigma))$ and $(x(0), y(0))=(1,0)$, and is the graph of a function $11=C_{(-1 / \sigma, 0)}(x)$ which is strictly decreasing and strictly convex on $(0,1)$.

Proof. The facts that $(x(-1 / \sigma), y(-1 / \sigma))=(0,1 /(e \sigma))$ and $(x(0), y(0))=(1,0)$ are obtained by direct substitution. Next, we may infer from the derivatives $x^{\prime}(\lambda)$ and $y^{\prime}(\lambda)$ that

and

$$
\frac{d y}{d x}=\lambda e^{(\sigma-\tau) \lambda}
$$

$$
\frac{d^{2} y}{d x^{2}}=\frac{1}{\tau} e^{(\sigma-2 \tau) \lambda} \frac{[1+(\sigma-\tau) \lambda]^{3}}{\sigma(\sigma-\tau) \lambda^{2}+(2 \sigma-\tau) \lambda+2},
$$

provided the denominator on the right-hand side is not zero. Since $\lambda \in(-1 / \sigma, 0)$ implies $\lambda e^{(\sigma-\tau) \lambda}<0$, we see that $C_{(-1 / \sigma, 0)}$ is strictly decreasing on $(0,1)$. We assert further that $d^{2} y / d x^{2}>0$ on the same interval. To see this, note that our assertion is clearly true when $\sigma=\tau$. When $\sigma>\tau$, we have $-1 /(\sigma-\tau)<-1 / \sigma$ and $1+(\sigma-\tau) \lambda>0$ for $\lambda \in(-1 / \sigma, 0)$. When $\sigma<\tau$, we also have $1+(\sigma-\tau) \lambda>0$ for $\lambda \in(-1 / \sigma, 0)$. Thus $[1+(\sigma-\tau) \lambda]^{3}>0$ for $\lambda \in(-1 / \sigma, 0)$. Next, consider the denominator in (7)):

$$
\phi(\lambda)=\sigma(\sigma-\tau) \lambda^{2}+(2 \sigma-\tau) \lambda+2,
$$

which has first and second derivatives

$$
\phi^{\prime}(\lambda)=2 \sigma(\sigma-\tau) \lambda+2 \sigma-\tau
$$

and

$$
\phi^{\prime \prime}(\lambda)=2 \sigma(\sigma-\tau)
$$

respectively. If $\sigma>\tau$, then $\phi^{\prime \prime}(\lambda)>0$ for $\lambda \in(-1 / \sigma, 0), \phi^{\prime}(-1 / \sigma)=\tau>0$ and $\phi(-1 / \sigma)=1$, so that $\phi(\lambda)>0$ for $\lambda \in(-1 / \sigma, 0)$. On the other hand, if $\sigma<\tau$, then $\lim _{\lambda \rightarrow-\infty} \phi(\lambda)=\lim _{\lambda \rightarrow \infty} \phi(\lambda)=-\infty, \phi(-1 / \sigma)=1>0$ and $\phi(0)=2>0$, so that $\phi(\lambda)>0$ for $\lambda \in(-1 / \sigma, 0)$ again. In view of (7), we see that $d^{2} y / d x^{2}>0$ for $x \in(0,1)$. The proof is complete.

The open region between the straight lines $x=0$ and $x=1$ and above the curve $C_{(-1 / \sigma, 0)}$ will be denoted by $\Omega$ :

$$
\Omega=\left\{(p, q) \mid 0<p<1, q>C_{(-1 / \sigma, 0)}(p)\right\} .
$$

If we rewrite (4) in the form

$$
-\lambda e^{-\tau \lambda} p+e^{-\sigma \lambda} q=-\lambda
$$

then we see that the point $(p, q)$ in the $x, y$-plane lies on the straight line

$$
-\lambda e^{-\tau \lambda} x+e^{-\sigma \lambda} y=-\lambda
$$

\footnotetext{
${ }^{1}$ We have used the same notation to denote a curve and also the function which describes it. This practice is convenient and will be followed in later proofs.
} 
if, and only if, $\lambda$ is a real solution of the characteristic equation

$$
h(\lambda ; p, q) \equiv \lambda-\lambda e^{-\tau \lambda} p+e^{-\sigma \lambda} q=0 .
$$

If we now interpret $h(\lambda ; x, y)=0$ as an equation describing a family $\Psi$ of straight lines of the form (8) with $\lambda$ as its parameter, then $h(\lambda ; p, q)=0$ for some real $\lambda$ if, and only if, there is a straight line from $\Psi$ passing through the point $(p, q)$ and tangent to its envelope.

The envelope $C$ of the family $\Psi$ is determined by the pair of equations

$$
h(\lambda ; x, y)=0
$$

and

$$
h^{\prime}(\lambda ; x, y)=1-x e^{-\tau \lambda}+x \lambda \tau e^{-\tau \lambda}-y \sigma e^{-\sigma \lambda}=0 .
$$

The corresponding pair of parametric equations $x=x(\lambda)$ and $y=y(\lambda)$ are easily obtained and given by (5).

We now consider four mutually exclusive and exhaustive cases: (I) $\tau=\sigma$, (II) $\tau>\sigma$, (III) $\sigma \geq \frac{1+\sqrt{2}}{2} \tau$, and (IV) $\tau<\sigma<\frac{1+\sqrt{2}}{2} \tau$.

CASE I. When $\tau=\sigma$, the parametric equations of the envelope are now

$$
\begin{aligned}
& x(\lambda)=e^{\sigma \lambda}(1+\sigma \lambda), \\
& y(\lambda)=e^{\sigma \lambda} \sigma \lambda^{2},
\end{aligned}
$$

for $\lambda \in R$, and (6) and (7) are now

$$
\frac{d y}{d x}=\lambda
$$

and

$$
\frac{d^{2} y}{d x^{2}}=\frac{1}{\sigma} e^{-\sigma \lambda} \frac{1}{\sigma \lambda+2},
$$

respectively. The graph of the envelope is easily sketched. To see this, we first use methods similar to those in the proof of Lemma 1 to show the following:

(a) $y(\lambda) \geq 0$ for $\lambda \in R$ and $y(\lambda)=0$ only if $\lambda=0$,

(b) $\lim _{\lambda \rightarrow-\infty} x(\lambda)=\lim _{\lambda \rightarrow-\infty} y(\lambda)=0$,

(c) $x(-1 / \sigma)=0, y(-1 / \sigma)=e^{\sigma(-1 / \sigma)} \sigma(-1 / \sigma)^{2}=1 /(e \sigma)$,

(d) $x(0)=1, y(0)=0$,

(e) $\lim _{\lambda \rightarrow+\infty} x(\lambda)=\lim _{\lambda \rightarrow+\infty} y(\lambda)=+\infty$,

(f) $d y / d x<0$ for $\lambda \in(-\infty,-2 / \sigma) \cup(-2 / \sigma, 0)$ and $d y / d x>0$ for $\lambda>0$,

(g) $d^{2} y / d x^{2}<0$ for $\lambda<-2 / \sigma$ and $d^{2} y / d x^{2}>0$ for $\lambda>-2 / \sigma$

These properties, together with other easily obtained information, allow us to depict the envelope $C$ of the family $\Psi$ in Figure 1 . The envelope $C$ is composed of two curves $C_{I}^{(1)}$ and $C_{I}^{(2)}$ and one turning point $\left(p^{*}, q^{*}\right)$. The turning point $\left(p^{*}, q^{*}\right)$ corresponds to $\lambda=-2 / \sigma$, i.e., $p^{*}=x(-2 / \sigma)=-1 / e^{2}$ and $q^{*}=y(-2 / \sigma)=4 / \sigma e^{2}$. The first piece $C_{I}^{(1)}$ corresponds to the case where $\lambda \in(-\infty,-2 / \sigma)$ and the second piece $C_{I}^{(2)}$ to the case where $\lambda \in(-2 / \sigma, \infty)$. Furthermore, the function $y=C_{I}^{(1)}(x)$ is strictly decreasing and strictly concave on $\left(p^{*}, 0\right)$, while the function $y=C_{I}^{(2)}(x)$ is strictly decreasing on $\left(p^{*}, 1\right)$, strictly increasing on $(1, \infty)$ and strictly convex for $x \in\left(p^{*}, \infty\right)$. The curve $C_{I}^{(2)}$ 


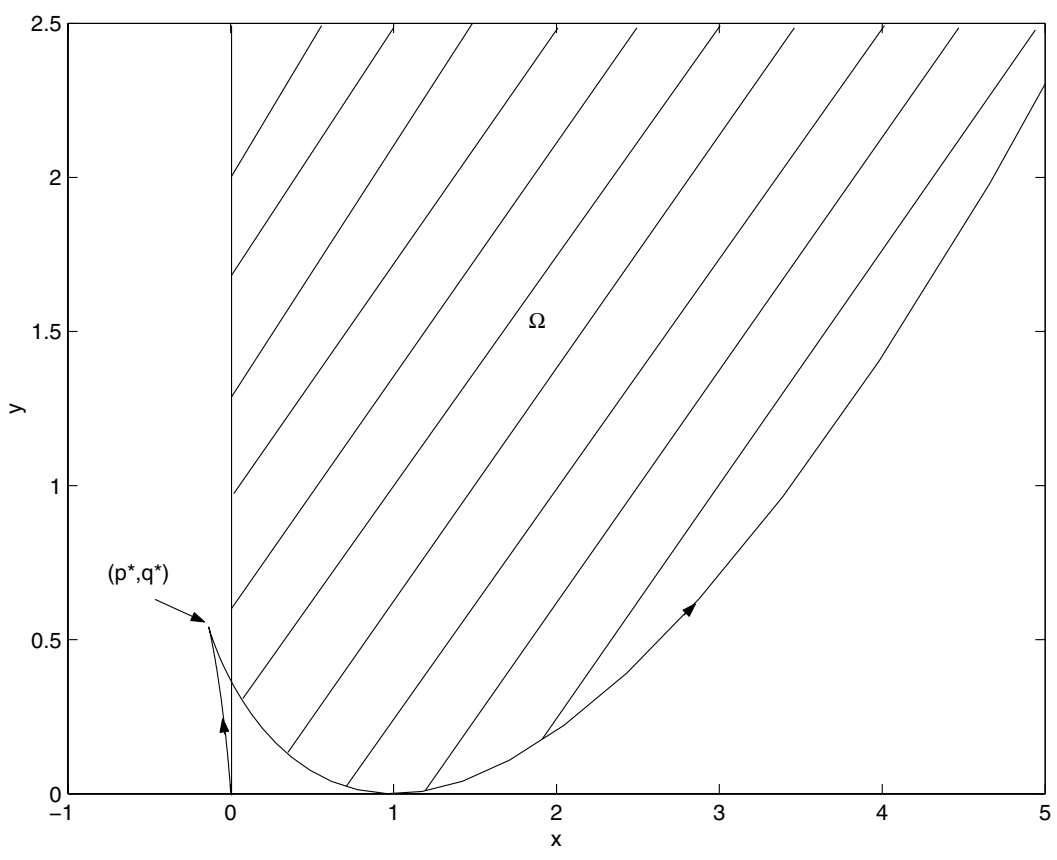

FIG. 1. $\sigma=\tau=1$

intersects the $y$-axis at the unique point $(0,1 /(e \sigma))$ which corresponds to $\lambda=-1 / \sigma$ and touches the $x$-axis at $(1,0)$ which corresponds to $\lambda=0$.

Let $\Omega_{I}$ be the set of all points in the plane which are located strictly above the curve $C_{I}^{(2)}$ and in the closed right half plane:

$$
\Omega_{I}=\left\{(p, q) \mid p \geq 0, q>C_{I}^{(2)}(p)\right\} .
$$

It is then easily seen that for each point $(p, q)$ contained in $\Omega_{I}$, there cannot be any tangent of the curve $C_{I}^{(1)}$ or the curve $C_{I}^{(2)}$ which also passes through this point, while for each point $(p, q)$ outside $\Omega_{I}$, such a tangent can be drawn. This statement needs a proof, but in view of Figure 1, it can be obtained by elementary means. Thus, in terms of the characteristic equation (44), the above observations imply that $h(\lambda ; \alpha, \beta)=0$ cannot have any real roots if, and only if, $(\alpha, \beta)$ belongs to $\Omega_{I}$.

CASE II. When $\tau>\sigma$, the graph of the envelope is easily sketched. To see this, we first note that the denominator $\phi(\lambda)=\sigma(\sigma-\tau) \lambda^{2}+(2 \sigma-\tau) \lambda+2$ in (77) has two real roots

$$
\lambda_{1}=\frac{-(2 \sigma-\tau)-\sqrt{\tau^{2}+4 \tau \sigma-4 \sigma^{2}}}{2 \sigma(\sigma-\tau)}
$$

and

$$
\lambda_{2}=\frac{-(2 \sigma-\tau)+\sqrt{\tau^{2}+4 \tau \sigma-4 \sigma^{2}}}{2 \sigma(\sigma-\tau)}
$$


which satisfies

$$
\lambda_{1}<0<\frac{1}{\tau-\sigma}<\lambda_{2}
$$

Then we may easily check that

(a) $\lim _{\lambda \rightarrow 1 /(\tau-\sigma)-0} x(\lambda)=\lim _{\lambda \rightarrow 1 /(\tau-\sigma)-0} y(\lambda)=+\infty$,

(b) $\lim _{\lambda \rightarrow 1 /(\tau-\sigma)+0} x(\lambda)=\lim _{\lambda \rightarrow 1 /(\tau-\sigma)+0} y(\lambda)=-\infty$,

(c) $\lim _{\lambda \rightarrow-\infty} x(\lambda)=\lim _{\lambda \rightarrow-\infty} y(\lambda)=0$,

(d) $x(-1 / \sigma)=0, y(-1 / \sigma)=1 / e \sigma$,

(e) $x(0)=1, y(0)=0$

(f) $\lim _{\lambda \rightarrow+\infty} x(\lambda)=\lim _{\lambda \rightarrow+\infty} y(\lambda)=-\infty$,

(g) $d y / d x<0$ for $\lambda \in\left(-\infty, \lambda_{1}\right) \cup\left(\lambda_{1}, 0\right)$ and $d y / d x>0$ for $\lambda \in(0,1 /(\tau-\sigma)) \cup$ $\left(1 /(\tau-\sigma), \lambda_{2}\right) \cup\left(\lambda_{2},+\infty\right)$,

(h) $d^{2} y / d x^{2}<0$ for $\lambda<\lambda_{1}$ and $\lambda \in\left(1 /(\tau-\sigma), \lambda_{2}\right)$,

(i) $d^{2} y / d x^{2}>0$ for $\lambda \in\left(\lambda_{1}, 1 /(\tau-\sigma)\right)$ and $\lambda>\lambda_{2}$,

(j) the unique asymptote of the envelope is described by the function

$$
y=\frac{1}{e(\tau-\sigma)}\left(x-e^{\tau /(\tau-\sigma)}\right),
$$

as can be seen from

$$
\lim _{x \rightarrow \pm \infty} \frac{C(x)}{x}=\lim _{\lambda \rightarrow 1 /(\tau-\sigma) \pm 0} \frac{y(\lambda)}{x(\lambda)}=\frac{1}{e(\tau-\sigma)}
$$

and

$\lim _{x \rightarrow \pm \infty}\left\{C(x)-\frac{1}{e(\tau-\sigma)} x\right\}=\lim _{\lambda \rightarrow 1 /(\tau-\sigma) \pm 0}\left\{y(\lambda)-\frac{1}{e(\tau-\sigma)} x(\lambda)\right\}=\frac{1}{e(\sigma-\tau)} e^{\tau /(\tau-\sigma)}$.

These properties, together with other easily obtained information, allow us to depict the envelope $C$ in Figure 2. The envelope $C$ is composed of four curves $C_{I I}^{(1)}, C_{I I}^{(2)}, C_{I I}^{(3)}, C_{I I}^{(4)}$ and two turning points $\left(p_{1}^{*}, q_{1}^{*}\right),\left(p_{2}^{*}, q_{2}^{*}\right)$. The turning points $\left(p_{1}^{*}, q_{1}^{*}\right)$ and $\left(p_{2}^{*}, q_{2}^{*}\right)$ correspond respectively to $\lambda_{1}$ and $\lambda_{2}$. The first piece $C_{I}^{(1)}$ corresponds to the case where $\lambda \in\left(-\infty, \lambda_{1}\right)$, the second piece to where $\lambda \in\left(\lambda_{1}, 1 /(\tau-\sigma)\right)$, the third to where $\lambda \in\left(1 /(\tau-\sigma), \lambda_{2}\right)$ and the fourth to where $\lambda \in\left(\lambda_{2}, \infty\right)$. Furthermore, the function $y=C_{I}^{(1)}(x)$ is strictly decreasing and strictly concave on $\left(p_{1}^{*}, 0\right)$, the function $y=C_{I}^{(2)}(x)$ is strictly decreasing on $\left(p_{1}^{*}, 1\right)$, strictly increasing on $(1, \infty)$ and strictly convex on $\left(p_{1}^{*}, \infty\right)$, the function $y=C_{I}^{(3)}(x)$ is strictly increasing and strictly concave on $\left(-\infty, p_{2}^{*}\right)$, and the function $y=C_{I}^{(4)}(x)$ is strictly increasing and strictly convex on $\left(-\infty, p_{2}^{*}\right)$.

As in the previous Case I, let $\Omega_{I I}$ be the set of all points in the plane which are located strictly above the curve $C_{I}^{(2)}$ and in the closed right half plane:

$$
\Omega_{I I}=\left\{(p, q) \mid p \geq 0, q>C_{I}^{(2)}(p)\right\} .
$$

Then it is easily seen that for each point $(p, q)$ contained in $\Omega_{I I}$, there cannot be any tangent of the curve $C_{I I}^{(1)}, C_{I I}^{(2)}, C_{I I}^{(3)}$ and $C_{I I}^{(4)}$ which also passes through this point, while for each point $(p, q)$ outside $\Omega_{I I}$, such a tangent can be drawn. Thus, in terms of the characteristic equation (4), the above observations imply that $h(\lambda ; \alpha, \beta)=0$ cannot have any real roots if, and only if, $(\alpha, \beta)$ belongs to $\Omega_{I I}$. 


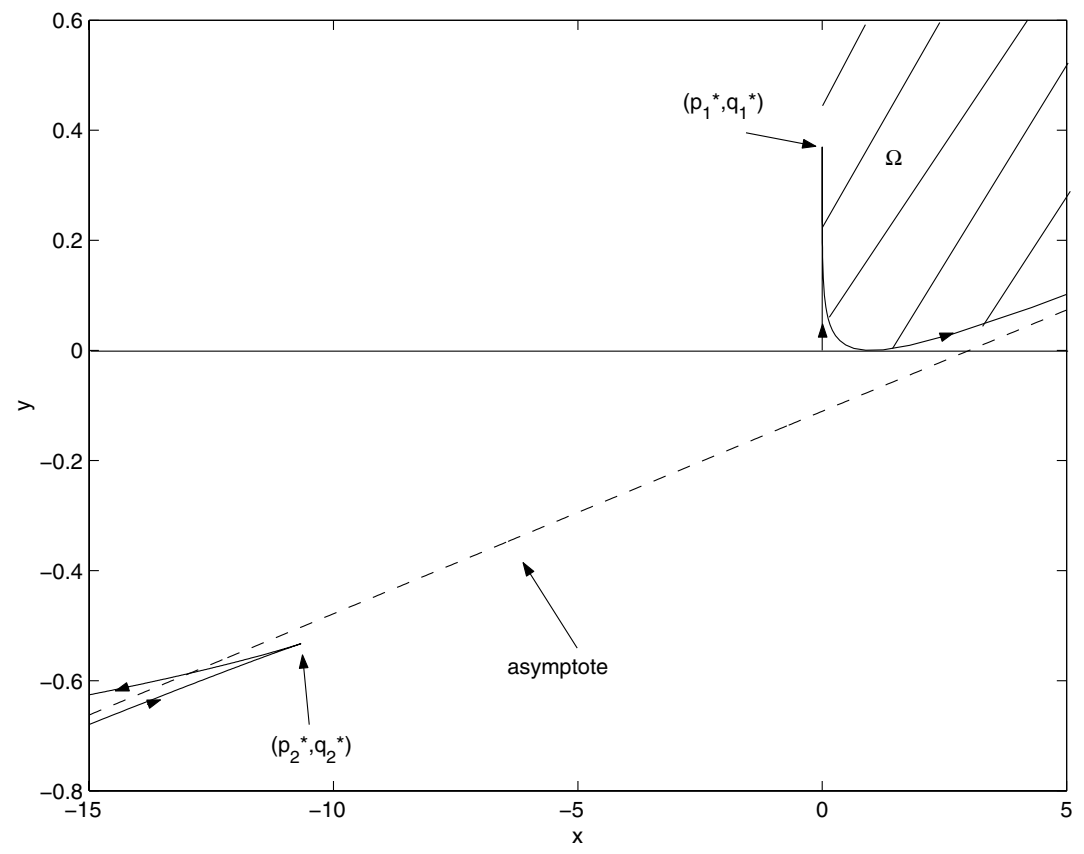

FIG. 2. $\sigma=1, \tau=11$

CASE III. When $\sigma \geq(1+\sqrt{2}) \tau / 2$, the graph of the envelope $C$ is easily sketched. To see this, first note that $\tau^{2}+4 \tau \sigma-4 \sigma^{2} \leq 0$. Thus the denominator $\phi(\lambda)=\sigma(\sigma-\tau) \lambda^{2}+$ $(2 \sigma-\tau) \lambda+2 \geq 0$ for $\lambda \in(-\infty, \infty)$ and may have a unique root $\lambda^{*}=(2 \sigma-\tau) / 2 \sigma(\tau-\sigma) \in$ $(-1 /(\sigma-\tau), 0)$. Then we may easily check that

(a) $\lim _{\lambda \rightarrow-1 /(\sigma-\tau)-0} x(\lambda)=+\infty, \lim _{\lambda \rightarrow-1 /(\sigma-\tau)-0} y(\lambda)=-\infty$,

(b) $\lim _{\lambda \rightarrow-1 /(\sigma-\tau)+0} x(\lambda)=-\infty, \lim _{\lambda \rightarrow-1 /(\sigma-\tau)+0} y(\lambda)=+\infty$,

(c) $\lim _{\lambda \rightarrow-\infty} x(\lambda)=\lim _{\lambda \rightarrow-\infty} y(\lambda)=0$,

(d) $x(-1 / \sigma)=0, y(-1 / \sigma)=1 / e \sigma$,

(e) $x(0)=1, y(0)=0$,

(f) $\lim _{\lambda \rightarrow+\infty} x(\lambda)=\lim _{\lambda \rightarrow+\infty} y(\lambda)=+\infty$,

(g) $d y / d x<0$ for $\lambda \in(-\infty,-1 /(\sigma-\tau))$ and $\lambda \in(-1 /(\sigma-\tau), 0)$, except possibly at $\lambda=\lambda^{*}$,

(h) $d y / d x>0$ for $\lambda>0$,

(i) $d^{2} y / d x^{2}<0$ for $\lambda<-1 /(\sigma-\tau)$ and $d^{2} y / d x^{2}>0$ for $\lambda>-1 /(\sigma-\tau)$, except possibly for $\lambda=\lambda^{*}$,

(j) the unique asymptote of the envelope $C$ is described by the function

$$
y=\frac{-1}{e(\sigma-\tau)}\left(x-e^{-\tau /(\sigma-\tau)}\right) .
$$

These properties together with other easily obtained information allow us to depict the envelope $C$ in Figure 3. The envelope $C$ is composed of two curves $C_{I I I}^{(1)}$ and $C_{I I I}^{(2)}$. The first curve corresponds to the case where $\lambda \in(-\infty,-1 /(\sigma-\tau))$ and the second to 


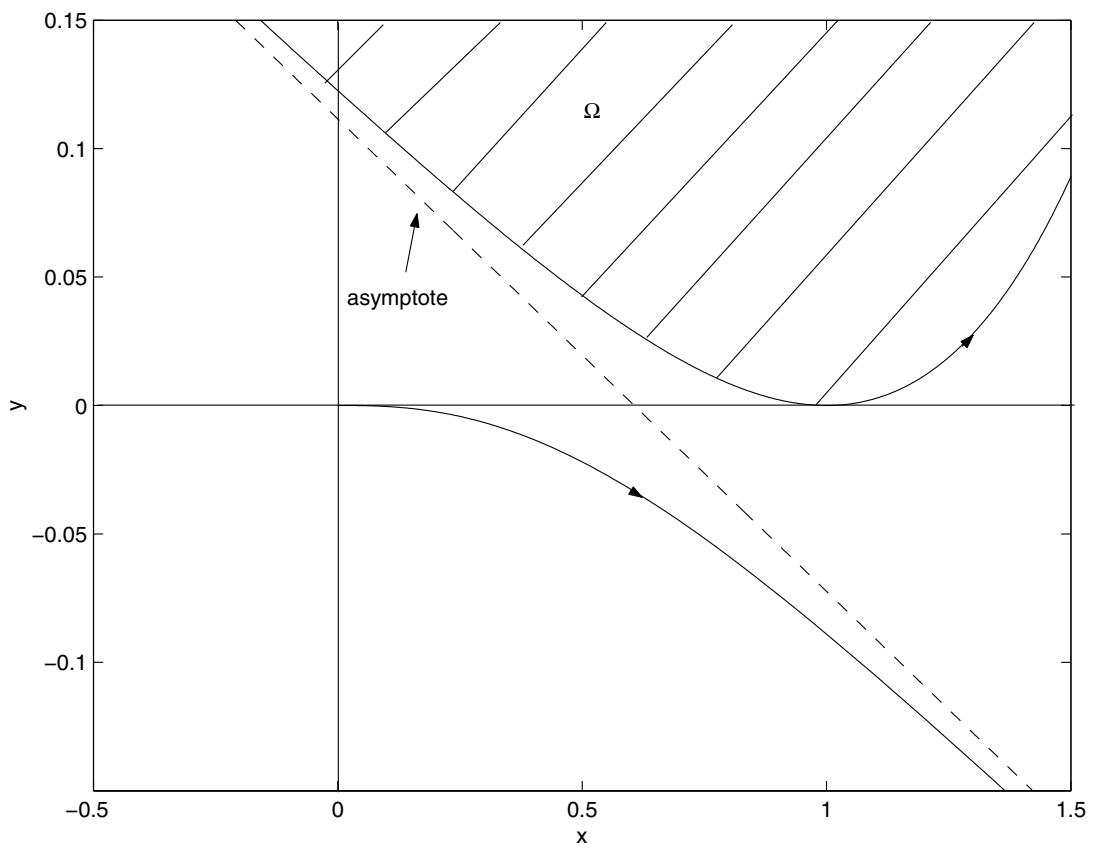

FIG. 3. $\sigma=3, \tau=1$

where $\lambda \in(-1 /(\sigma-\tau), \infty)$. Furthermore, the function $y=C_{I I I}^{(1)}(x)$ is strictly negative, strictly decreasing and strictly concave on $(0, \infty)$, and the function $y=C_{I I I}^{(2)}(x)$ is strictly decreasing on $(0,1)$, strictly increasing on $(1, \infty)$ and strictly convex on $(0, \infty)$.

As in the previous Case I, let $\Omega_{I I I}$ be the set of all points in the plane which are located strictly above the curve $C_{I I I}^{(2)}$ :

$$
\Omega_{I I I}=\left\{(p, q) \mid q>C_{I I I}^{(2)}(p)\right\} .
$$

Then it is easily seen that for each point $(p, q)$ contained in $\Omega_{I I I}$, there cannot be any tangent of the curve $C_{I I I}^{(1)}$ or the curve $C_{I I I}^{(2)}$ which also passes through this point, while for each point $(p, q)$ outside $\Omega_{I I I}$, such a tangent can be drawn. Thus, in terms of the characteristic equation (4), the above observations imply that $h(\lambda ; \alpha, \beta)=0$ cannot have any real roots if, and only if, $(\alpha, \beta)$ belongs to $\Omega_{I I I}$.

CASE IV. When $\tau<\sigma<(1+\sqrt{2}) \tau / 2$, the graph of the envelope $C$ is easily sketched. To see this, first note that $\tau^{2}+4 \tau \sigma-4 \sigma^{2}>0$. Thus the denominator

$$
\phi(\lambda)=\sigma(\sigma-\tau) \lambda^{2}+(2 \sigma-\tau) \lambda+2
$$

has two real roots

$$
\lambda_{1}=-\frac{2 \sigma-\tau}{2 \sigma(\sigma-\tau)}-\frac{\sqrt{\tau^{2}+4 \tau \sigma-4 \sigma^{2}}}{2 \sigma(\sigma-\tau)}
$$

and

$$
\lambda_{2}=-\frac{2 \sigma-\tau}{2 \sigma(\sigma-\tau)}+\frac{\sqrt{\tau^{2}+4 \tau \sigma-4 \sigma^{2}}}{2 \sigma(\sigma-\tau)},
$$




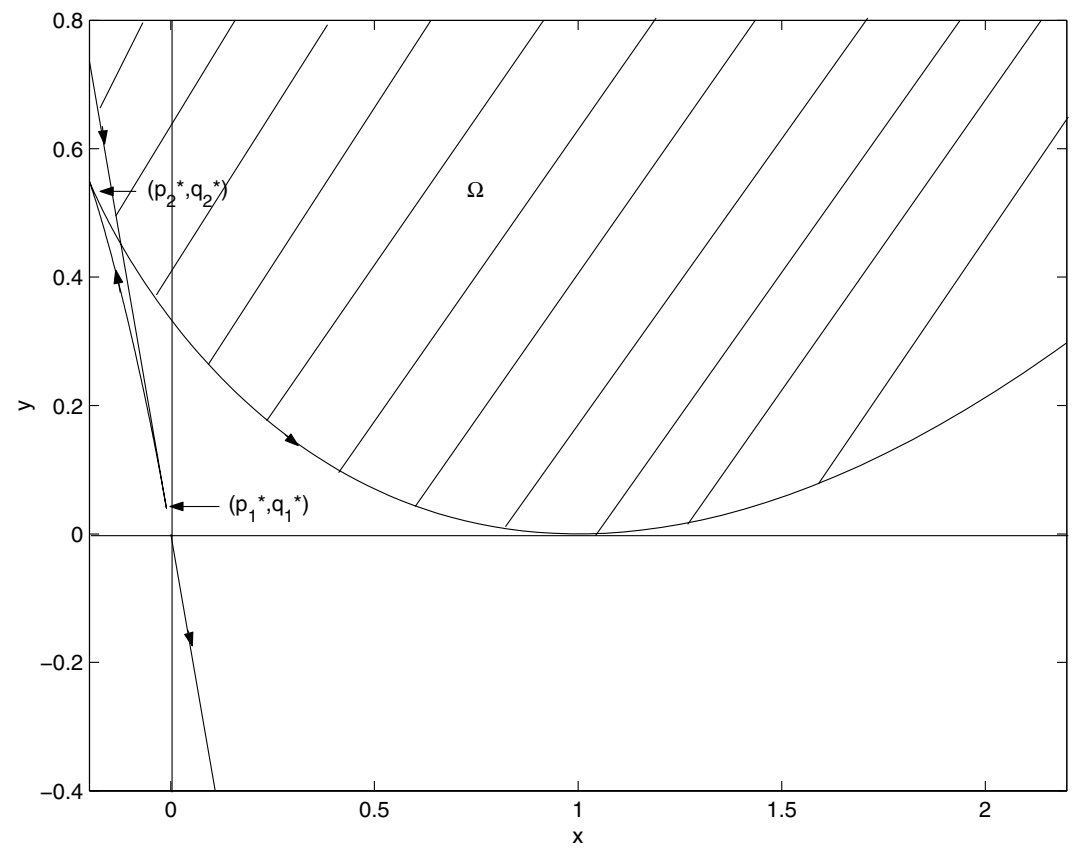

FIG. 4. $\sigma=1.1, \tau=1$

which satisfy

$$
\frac{-1}{\sigma-\tau}<\lambda_{1}<\lambda_{2}<\frac{-1}{\sigma}<0 .
$$

Then we may easily check that

(a) $\lim _{\lambda \rightarrow-1 /(\sigma-\tau)-0} x(\lambda)=+\infty, \lim _{\lambda \rightarrow-1 /(\sigma-\tau)-0} y(\lambda)=-\infty$,

(b) $\lim _{\lambda \rightarrow-1 /(\sigma-\tau)+0} x(\lambda)=-\infty, \lim _{\lambda \rightarrow-1 /(\sigma-\tau)+0} y(\lambda)=+\infty$,

(c) $\lim _{\lambda \rightarrow-\infty} x(\lambda)=\lim _{\lambda \rightarrow-\infty} y(\lambda)=0$,

(d) $x(-1 / \sigma)=0, y(-1 / \sigma)=1 / e \sigma$,

(e) $x(0)=1, y(0)=0$,

(f) $d y / d x<0$ for $\lambda \in(-\infty,-1 /(\sigma-\tau)) \cup\left(-1 /(\sigma-\tau), \lambda_{1}\right) \cup\left(\lambda_{1}, \lambda_{2}\right) \cup\left(\lambda_{2}, 0\right)$ and $d y / d x>0$ for $\lambda>0$,

(g) $d^{2} y / d x^{2}<0$ for $\lambda \in(-\infty,-1 /(\sigma-\tau)) \cup\left(\lambda_{1}, \lambda_{2}\right)$ and $d^{2} y / d x^{2}>0$ for $\lambda \in$ $\left(-1 /(\sigma-\tau), \lambda_{1}\right) \cup\left(\lambda_{2},+\infty\right)$,

(h) the asymptote of the envelope $C$ is described by the function

$$
y=\frac{-1}{e(\sigma-\tau)}\left(x-e^{-\tau /(\sigma-\tau)}\right) .
$$

These properties, together with other easily obtained information, allow us to depict the envelope $C$ in Figure 4. The envelope $C$ is composed of four curves $C_{I V}^{(1)}, C_{I V}^{(2)}, C_{I V}^{(3)}$, $C_{I V}^{(4)}$ and two turning points $\left(p_{1}^{*}, q_{1}^{*}\right),\left(p_{2}^{*}, q_{2}^{*}\right)$. The turning points $\left(p_{1}^{*}, q_{1}^{*}\right)$ and $\left(p_{2}^{*}, q_{2}^{*}\right)$ correspond respectively to $\lambda_{1}$ and $\lambda_{2}$, that is, $p_{1}^{*}=x\left(\lambda_{1}\right), q_{1}^{*}=y\left(\lambda_{1}\right), p_{2}^{*}=x\left(\lambda_{2}\right)$ and $q_{2}^{*}=x\left(\lambda_{2}\right)$. The first piece corresponds to the case where $\lambda \in(-\infty,-1 /(\sigma-\tau))$, the 
second to where $\lambda \in\left(-1 /(\sigma-\tau), \lambda_{1}\right)$, the third to where $\lambda \in\left(\lambda_{1}, \lambda_{2}\right)$, and the fourth to where $\lambda \in\left(\lambda_{2},+\infty\right)$. Furthermore, the function $y=C_{I V}^{(1)}(x)$ is strictly negative, strictly decreasing and strictly concave on $(0,+\infty)$, the function $y=C_{I V}^{(2)}(x)$ is strictly decreasing and strictly convex on $\left(-\infty, p_{1}^{*}\right)$, the function $y=C_{I V}^{(3)}(x)$ is strictly decreasing and strictly concave on $\left(p_{2}^{*}, p_{1}^{*}\right)$, and the function $y=C_{I V}^{(4)}(x)$ is strictly decreasing and strictly convex on $\left(p_{2}^{*},+\infty\right)$.

As in the previous Case I, let $\Omega_{I V}$ be the set of all points in the plane which are located strictly above the curve $C_{I V}^{(2)}$ and strictly above the curve $C_{I V}^{(4)}$ :

$$
\Omega_{I V}=\left\{(p, q) \mid q>C_{I V}^{(2)}(p), q>C_{I V}^{(4)}(p)\right\} .
$$

Then it is easily seen that for each point $(p, q)$ contained in $\Omega_{I V}$, there cannot be any tangent of the curves $C_{I V}^{(1)}, C_{I V}^{(2)}, C_{I V}^{(3)}$ and $C_{I V}^{(4)}$ which also passes through this point, while for each point $(p, q)$ outside $\Omega_{I V}$, such a tangent can be drawn. Thus, in terms of the characteristic equation (4), the above observations imply that $h(\lambda ; \alpha, \beta)=0$ cannot have any real roots if, and only if, $(\alpha, \beta)$ belongs to $\Omega_{I V}$.

THEOREM 1. Every solution of (3) oscillates if, and only if, one of the following conditions hold:

(I) $\tau=\sigma,(p, q) \in \Omega_{I}$

(II) $\tau>\sigma,(p, q) \in \Omega_{I I}$;

(III) $\sigma \geq \frac{1+\sqrt{2}}{2} \tau,(p, q) \in \Omega_{I I I}$;

(IV) $\tau<\sigma<\frac{1+\sqrt{2}}{2} \tau,(p, q) \in \Omega_{I V}$.

Note that $\Omega$ is contained in $\Omega_{I}, \Omega_{I I}, \Omega_{I I I}$ and $\Omega_{I V}$, and its relative complement in $\{(p, q) \mid 0<p<1\}$ is contained in the complements of $\Omega_{I}, \Omega_{I I}, \Omega_{I I I}$ and $\Omega_{I V}$. Hence we have the following.

Corollary 1. Under the condition that $p \in(0,1)$, every solution of (3) oscillates if, and only if, $(p, q) \in \Omega$.

3. Explicit and sharp conditions. We have mentioned that our geometric necessary and sufficient condition can provide explicit and sharp oscillation criteria. We illustrate this by considering the case where $p \in(0,1)$ by making use of the convexity of the curve $C_{(-1 / \sigma, 0)}$ (see Lemma 1) to find explicit conditions for (3) to be oscillatory.

Before doing so, we remark that since the function $x(\lambda)$ in (5) is strictly increasing over the interval $(-1 / \sigma, 0)$, for each $p \in(0,1)$, there is a unique $\kappa \in(-1 / \sigma, 0)$ such that $p=x(\kappa)$. It follows that when $p \in(0,1),(p, q) \in \Omega$ if, and only if, $q>y(\kappa)$.

Let us now connect the endpoints $A=(0,1 /(e \sigma))$ and $B=(1,0)$ of the curve $C_{(-1 / \sigma, 0)}$ by a straight line segment; then this line segment is described by

$$
L_{1}(x)=\frac{1}{e \sigma}(1-x), x \in(0,1) .
$$

Since the function $y=C_{(-1 / \sigma, 0)}(x)$ is strictly convex on $(0,1)$, this line segment is above the curve $C_{(-1 / \sigma, 0)}$. Thus for any $(p, q)$ which satisfies $0<p<1$ and $q \geq L_{1}(p)=$ $(1-p) /(e \sigma)$, every solution of (3) is oscillatory. This set of conditions is sharp, for when 
$p=0$ and $q=(1-0) /(e \sigma)=1 /(e \sigma)$, equation (3) has a nonoscillatory solution in view of Theorem 1.

Next, note that the slopes of the curve $C_{(-1 / \sigma, 0)}$ through the endpoints $(0,1 /(e \sigma))$ and $(1,0)$ are $-e^{\tau / \sigma} /(e \sigma)$ and 0 , respectively. Thus the tangent lines through these two endpoints are described by

$$
L_{2}(x)=\frac{1}{e \sigma}-\frac{1}{e \sigma} e^{\tau / \sigma} x, x \in R,
$$

and

$$
L_{3}(x)=0, x \in R .
$$

The point of intersection of these two straight lines is $\left(e^{-\tau / \sigma}, 0\right)$. By means of the convexity of the curve $C_{(-1 / \sigma, 0)}$, we thus see that the graph of the function

$$
h_{1}(x)= \begin{cases}L_{2}(x), & 0<x \leq e^{-\tau / \sigma} \\ 0, & e^{-\tau / \sigma} \leq x<1\end{cases}
$$

is situated below the curve $C_{(-1 / \sigma, 0)}$. In view of Theorem 1 , if $(p, q)$ satisfies $p \in(0,1)$ and $q \leq h_{1}(p)$, then (3) has a nonoscillatory solution. This condition is sharp since when $p=0$ and $q>h_{1}(0)=1 /(e \sigma)$, (3) is oscillatory.

Theorem 2. If $(p, q)$ satisfies $p \in(0,1)$ and $q \geq(1-p) /(e \sigma)$, then every solution of (3) is oscillatory. If $(p, q)$ satisfies $p \in(0,1)$ and

$$
q \leq \begin{cases}\frac{1}{e \sigma}\left(1-e^{\tau / \sigma} p\right), & 0<p \leq e^{-\tau / \sigma}, \\ 0, & e^{-\tau / \sigma} \leq p<1,\end{cases}
$$

then (3) has a nonoscillatory solution.

Next, let us repeat the same ideas by picking the midpoint $-1 /(2 \sigma)$ of the interval $(-1 / \sigma, 0)$. The corresponding point in the graph $C_{(-1 / \sigma, 0)}$ is

$$
D=\left(\frac{\sigma}{\sigma+\tau} e^{-\tau /(2 \sigma)}, \frac{\tau}{2 \sigma(\sigma+\tau)} e^{-1 / 2}\right) .
$$

The straight line segment connecting the point $A=(0,1 /(e \sigma))$ and $D$ is described by

$$
y=\frac{1}{e \sigma}-\frac{2(\sigma+\tau)-\tau e^{1 / 2}}{2 e \sigma^{2}} e^{\tau /(2 \sigma)} x, 0<x \leq \frac{\sigma}{\sigma+\tau} e^{-\tau /(2 \sigma)},
$$

while the straight line segment connecting $D$ and $B=(1,0)$ is described by

$$
y=\frac{\tau}{2 \sigma\left[(\sigma+\tau) e^{1 / 2}-\sigma e^{(\sigma-\tau) / 2 \tau}\right]}(1-x), \frac{\sigma}{\sigma+\tau} e^{-\tau /(2 \sigma)} \leq x<1 .
$$

In view of the convexity of $C_{(-1 / \sigma, 0)}$, the above line segments lie above $C_{(-1 / \sigma, 0)}$ except at the point $D$. By Theorem 1 , we see that if $(p, q)$ satisfies $p \in(0,1)$ and

$$
q \begin{cases}\geq \frac{1}{e \sigma}-\frac{2(\sigma+\tau)-\tau e^{1 / 2}}{2 e \sigma^{2}} e^{\tau /(2 \sigma)} p, & 0<p<\frac{\sigma}{\sigma+\tau} e^{-\tau /(2 \sigma),}, \\ \geq \frac{\tau}{2 \sigma\left[(\sigma+\tau) e^{1 / 2}-\sigma e^{(\sigma-\tau) / 2 \tau}\right]}(1-p), & \frac{\sigma}{\sigma+\tau} e^{-\tau /(2 \sigma)}<p<1, \\ >\frac{\tau}{2 \sigma(\sigma+\tau)} e^{-1 / 2}, & p=\frac{\sigma}{\sigma+\tau} e^{-\tau /(2 \sigma)},\end{cases}
$$

then every solution of (3) is oscillatory. This condition is sharp. 
On the other hand, since the tangent of the curve $C_{(-1 / \sigma, 0)}$ through the point $D$ is described by

$$
L_{4}(x)=\frac{1}{2 \sigma e^{1 / 2}}\left(1-e^{\tau /(2 \sigma)} x\right), x \in R,
$$

and since the $x$-coordinate of the point of intersection of the lines $L_{2}$ and $L_{4}$ is

$$
x^{*}=\frac{2-\sqrt{e}}{2 e^{\tau / \sigma}-e^{1 / 2} e^{\tau /(2 \sigma)}},
$$

while the point of intersection of the lines $L_{4}$ and $L_{3}$ is $\left(e^{-\tau /(2 \sigma)}, 0\right)$, thus by Theorem 1 , when $(p, q)$ satisfies $p \in(0,1)$ and

$$
q \leq \begin{cases}\frac{1}{e \sigma}\left(1-e^{\tau / \sigma} p\right), & 0<p \leq x^{*} \\ \frac{1}{2 \sigma e^{1 / 2}}\left(1-e^{\tau /(2 \sigma)} p\right), & x^{*} \leq p \leq e^{-\tau /(2 \sigma)} \\ 0, & e^{-\tau /(2 \sigma)} \leq p<1\end{cases}
$$

then (3) has a nonoscillatory solution. This condition is sharp.

By picking distinct points $\lambda_{1}, \lambda_{2}, \ldots, \lambda_{n}$ in the interval $(-1 / \sigma, 0)$, we may follow the same principles above and obtain better oscillation criteria. Since there are no new principles involved, we will only state the corresponding result when $\lambda_{1}=-3 / 4 \sigma, \lambda_{2}=$ $-1 / 2 \sigma$ and $\lambda_{3}=-1 / 4 \sigma$.

Theorem 3. If $(p, q)$ satisfies $p \in(0,1)$ and

$$
q \begin{cases}\geq \frac{1}{e \sigma}+\left(\frac{q \tau e^{1 / 4}-4 \sigma-12 \tau}{4 \sigma^{2} e^{1-3 \tau / 4 \sigma}}\right) p, & 0<p<x_{1}, \\ >\frac{\chi \tau e^{-3 / 4}}{4 \sigma^{2}+12 \sigma \tau}, & p=x_{1}, \\ \geq \frac{\left(2 \sigma \tau+6 \tau^{2}\right) e^{-1 / 2}-\left(9 \tau^{2}+96 \tau\right) e^{-3 / 4}}{\left(12 \sigma^{2} \tau+4 \sigma^{3}\right) e^{-\tau / 2 \sigma}-\left(4 \sigma^{3}+4 \sigma^{2} \tau\right) e^{-3 \tau / 4 \sigma}}\left(p-\frac{\sigma}{\sigma+\tau} e^{-\tau / 2 \sigma}\right)+\frac{\tau e^{-1 / 2}}{2 \sigma(\sigma+\tau)}, & x_{1}<p<x_{2}, \\ >\frac{\tau e^{-1 / 2}}{2 \sigma^{2}+2 \sigma \tau}, & p=x_{2}, \\ \geq \frac{\left(\sigma \tau+\tau^{2}\right) e^{-1 / 4}-\left(2 \tau^{2}+6 \sigma \tau\right) e^{-1 / 4}}{\left(12 \sigma^{3}+12 \sigma^{2} \tau\right) e^{-\tau / 4 \sigma}-\left(12 \sigma^{3}+4 \sigma^{2} \tau\right) e^{-\tau / 2 \sigma}}\left(p-\frac{3 \sigma}{3 \sigma+\tau} e^{-\tau / 4 \sigma}\right)+\frac{\tau e^{-1 / 4}}{12 \sigma^{2}+4 \sigma \tau}, & x_{2}<p<x_{3}, \\ >\frac{\tau e^{-1 / 4}}{12 \sigma^{2}+4 \sigma \tau}, & p=x_{3}, \\ \geq \frac{\tau e^{-1 / 4}}{12 \sigma^{2} e^{-\tau / 4 \sigma}-12 \sigma^{2}-4 \sigma \tau}(p-1), & x_{3}<p<1,\end{cases}
$$

where

$$
x_{1}=\frac{\sigma}{\sigma+3 \tau} e^{-3 \tau /(4 \sigma)}, x_{2}=\frac{\sigma}{\sigma+\tau} e^{-\tau /(2 \sigma)}, x_{3}=\frac{3 \sigma}{3 \sigma+\tau} e^{-\tau / 4 \sigma},
$$

then every solution of (3) is oscillatory. If $(p, q)$ satisfies $p \in(0,1)$ and

$$
q \leq \begin{cases}\frac{1}{e \sigma}\left(1-e^{\tau / \sigma} p\right), & 0<p \leq x_{1}^{*} \\ \frac{3}{4 \sigma e^{3 / 4}}\left(1-e^{3 \tau /(4 \sigma)} p\right), & x_{1}^{*} \leq p \leq x_{2}^{*} \\ \frac{1}{2 \sigma e^{1 / 2}}\left(1-e^{\tau /(2 \sigma)} p\right), & x_{2}^{*} \leq p \leq x_{3}^{*} \\ \frac{1}{4 \sigma e^{1 / 4}}\left(1-e^{\tau /(4 \sigma)} p\right), & x_{3}^{*} \leq p \leq x_{4}^{*} \\ 0, & x_{4}^{*} \leq p<1\end{cases}
$$

where

$$
\begin{gathered}
x_{1}^{*}=e^{-3 \tau /(4 \sigma)} \frac{4-3 e^{1 / 4}}{4 e^{\tau /(4 \sigma)}-3 e^{1 / 4}}, x_{2}^{*}=e^{-\tau / 2 \sigma} \frac{3-2 e^{1 / 4}}{3 e^{\tau /(4 \sigma)}-2 e^{1 / 4}}, \\
x_{3}^{*}=e^{-\tau /(4 \sigma)} \frac{2-e^{1 / 4}}{2 e^{\tau /(4 \sigma)}-e^{1 / 4}}, x_{4}^{*}=e^{-\tau /(4 \sigma)},
\end{gathered}
$$

then (3) has a nonoscillatory solution. 
As our final remark, we quote a recent result of Lin [5] which states that if $p \in(0,1)$ and

$$
q \sigma e>1-p e^{q \tau /(1-p-q \sigma)},
$$

then every solution of (3) oscillates. In view of our Corollary 1, we may assert that

$$
\begin{aligned}
& \left\{(p, q) \in R^{2} \mid 0<p<1, q \sigma e>1-p e^{q \tau /(1-p-q \sigma)}\right\} \\
& \subset\left\{(p, q) \in R^{2} \mid 0<p<1, q>C_{(-1 / \sigma, 0)}(p)\right\},
\end{aligned}
$$

a statement which may be difficult to prove directly since (10) is a functional inequality.

\section{REFERENCES}

[1] H. S. Ren and Z. X. Zheng, The algebraic criteria of oscillation of linear neutral differential equations with delays, J. Biomath., 13(1)(1998), 43-46 (in Chinese). MR.1845813 (2002b:34107)

[2] H. S. Ren, On the accurate distribution of characteristic roots and stability of linear delay differential systems, Northeastern Forestry University Press, Harbin, 1999 (in Chinese).

[3] S. S. Cheng and Y. Z. Lin, Exact regions of oscillation for a neutral differential equation, Proc. Royal Soc. Edin., 130A(2000), 277-286. MR.1750831 (2001k:34125)

[4] V. G. Boltyanskii, Envelopes, Popular Lectures in Mathematics, Vol. 12, Macmillan Company, New York, 1964.

[5] S. Z. Lin, Oscillation in first order neutral differential equations, Ann. Diff. Eqs., 19(3)(2003), 334-336. MR:2018300 (2005g:34159)

[6] I. Györi and G. Ladas, Oscillation Theory of Delay Differential Equations, Oxford Science Publications, Clarendon Press, Oxford, 1991. MR:1168471 (93m:34109) 